\title{
LA EDUCACIÓN SOCIAL EN LOS CENTROS EDUCATIVOS INSTITUCIONALIZADOS POSMODERNOS
}

\author{
Monia Rodorigo \\ Tamara Aguirre-Martín \\ Universidad de Almería
}

\begin{abstract}
RESUMEN: Las características y valores de la sociedad requieren repensar la acción educativa. Múltiples son los elementos a modificar, pero uno de ellos es sin duda representado por el debate pedagógico que subyace a la incorporación de la Educación Social en las escuelas. Así, partiendo de una revisión teórica sobre las características y valores imperantes en la sociedad, y llevando a cabo una investigación bibliográfica sobre el papel del Educador o Educadora Social en las escuelas, este artículo reflexiona sobre la necesidad de la Educación Social en la formación de la moral posmoderna; analizando epistemológicamente las bases sobre las que se ejecuta y el marco desde el que se enfoca. Asimismo, se plantea la necesidad de -junto con la entrada de estos profesionales- repensar el para qué de las escuelas actuales rompiendo con la asignaturización del acto educativo, subrayando la necesidad de entender la tarea docente como holística y colaborativa, en aras de responder a las necesidades posmodernas actuales.
\end{abstract}

PALABRAS CLAVE: educación social, posmodernidad, centros educativos institucionalizados, funciones, necesidades sociales.

\section{THE SOCIAL EDUCATION IN POSMODERN AND INSTITUTIONALIZED EDUCATIONAL CENTERS}

\begin{abstract}
The specific characteristics and the reference values of the society require to rethink educational action. It is necessary to modify a lot of stuff but, due to the pedagogical debate, we have to mention the social education necessity into schools. Starting with a theoretical revision about postmodern society and carried out a bibliography research is justified that social educator had to work there, because of the important role he assumes on comprehensive education and on student's postmodern morality. We describe their assumed role, their task and their role. We also consider the possibility
\end{abstract}


of rethink the main goal of education system, breaking traditional schemes in favor of a holistic and collaborative teacher's activity and pedagogy with closer connection with postmodern society.

KEYWORDS: Social education, postmodernity, educational centers, roles.

Recibido: 02/07/2018

Aceptado: 11/01/2019

Correspondencia: Monia Rodorigo, Facultad de Educación, Universidad de Almería. Ctra. Sacramento s/n. La Cañada de San Urbano, 04120 Almería. Email: mrodorigo@ual.es.

\section{INTRODUCCIÓN, O DE LA EDUCACIÓN SOCIAL POSMODERNA}

La acentuación por lo individual, la satisfacción inmediata de necesidades establecidas por un mercado dominante, el predominio de lo efímero ante un futuro incierto, la alteración de los conceptos y paradigmas a los que la ciudadanía se acoge, la creación de nuevas demandas sociales que se asimilan como incuestionables y que lejos de ser repensadas como base del debate social acaban conformando el discurso hegemónico y por ende el sentido común y la identidad cultural; son algunas de las características que definen hoy en día la sociedad.

Una sociedad líquida (Bauman, 2003) posmoderna y de servicios que genera espacios cambiantes caracterizados por el cuestionamiento de todo lo anterior, de los grandes metarrelatos (Lyotard, 1979) y de las narrativas que habían definido la verdad en la época moderna. Se trasforman las estructuras sociales, que pasan a ser invadidas por constantes cambios y la desaparición de los límites de la sociabilidad, problematiza a su vez, las relaciones individuo-comunidad alterando los espacios comunitarios.

Los actores sociales son individuos narcisistas que Lipovetsky (2003) define no como seres egocéntricos triunfantes, sino, como individuos frágiles, llamados a construirse sin normas sociales en las que apoyarse. La ética se desvirtúa. La moral sobre la que se fundamenta es hoy día "una moral interpersonal y emocional, indolora y no imperativa, adaptada a los nuevos valores de autonomía individualista" (2003, p. 44), ya que "la cultura de los deberes relativos a uno mismo ha sido sustituida por la de los derechos individualistas..." (2003, pp. 39-40) según los que el individuo, ya no se debe a la sociedad sino a sí mismo. La estructura económica también se ha visto modificada. El mercado se ha vuelto soberano y la ética se ha visto sobrepasada por la eficacia. El conocimiento se ha trasformado en baza productiva, por lo que se ha convertido en una moneda de cambio que acaba definiendo las posibilidades y oportunidades de la ciudadanía en función de su alcance o acceso a la misma información.

En este escenario la creación de consciencias regulares que sean capaces de asumir, más allá de la paradoja postmoderna (Lyotard, 1979) un individualismo basado 
en la responsabilidad y la solidaridad; y el pensamiento crítico, entendido como una herramienta de libertad y formación de la ciudadanía, capaz de promover el aprendizaje y la interiorización de las percepciones para la reconstrucción social; cobran cada vez más sentido.

La educación se transforma en un espacio privilegiado para las tareas encomendadas a la ciudadanía. Solo a través de la educación en general y de la educación social -entendida como promotora de cambio social- en particular, conseguiremos preparar a la ciudadanía para este cambio conceptual que, indudablemente, ya ha modificado las estructuras sociales, pero que sin embargo, todavía no ha calado a la hora de replantearse el para qué del acto educativo. Hablar de educación ya no sólo significa hablar de instrucción, sino que es necesario repensar la acción de la escuela como espacio ecológico de cambio en el que el alumnado posmoderno reconstruya su identidad sobre la base de una conciencia de responsabilidad pública que ayude a su vez a mitigar las desigualdades sociales.

Necesitamos de unos profesionales, que junto con maestros y maestras, psicopedagogos y psicopedagogas, mediadores y mediadoras interculturales sean capaces de trabajar para el desarrollo integral de niños y niñas cuidando

los procesos y las dinámicas socioeducativas que posibilitan y facilitan el óptimo desarrollo de los procesos de socialización, tanto desde una perspectiva inespecífica -aquellos procesos y aquellas dinámicas socioeducativas indiferenciadas que afectan a cualquier persona en cualquier espacio socialcomo desde una perspectiva específica -aquellos procesos y aquellas dinámicas socioeducativas diferenciadas que principalmente tienen lugar dentro de una modalidad de la educación no formal y que básicamente, aunque no exclusivamente, afectan a personas que presentan necesidades particulares respecto a sus procesos de integración social (Gómez-Serra, 2003, p. 235).

Unos profesionales capaces de impulsar desde una perspectiva inespecífica la "revolución cultural" que Bauman (2013) propone; y abordar, desde una perspectiva específica, con y para los colectivos vulnerables o anormales ${ }^{1}$, una acción generadora que permita la inclusión social, o como señalaría Freire (2012), con objetivo de crear una situación que libere al oprimido, liberando al mismo tiempo al opresor en esta compleja época posmoderna.

Por todo ello, a lo largo de este trabajo y haciendo un recorrido por las claves ontológicas, epistemológicas y metodológicas sobre las que se construye la Educación Social intentaremos profundizar en los siguientes propósitos/objetivos:

1) analizar el papel y las funciones que asume o debería de asumir el educador social en la escuela como agente de cambio y mejora;

2) conocer las posibilidades de acción del educador social en este entorno, teniendo en cuenta especialmente: a) las relaciones interpersonales que se establecen y b) la estructura organizativo-espacial de las instituciones educativas;

1. Entiéndase anormal, desde la distinción que hace Bauman (2013) de aquellos que se alejan de la norma. 


\section{Repensar epistemológicamente la aCCiÓn EDUCATIVA en la era POSMODERNA, O DE LA NECESIDAD DE LA EDUCACIÓN SOCIAL EN LA ESCUELA}

En un contexto como el explicado anteriormente, la acción educativa necesita de una re-estructuración conceptual desde la que se analice el concepto de educación desde su perspectiva más amplia, sin que esta se vea subordinada al espacio en el que se desarrolla su praxis o a la especialización de los profesionales que la guían.

Separar el proceso instructivo del educativo, como se ha hecho históricamente, o definir como formal o no formal la acción educativa de distintos profesionales a pesar de lo que distintos autores han hecho presente "si algo es educación, es formal; si es no formal o es informal, no es educación"”2 (Ortega, 2005, p. 168); ha permitido que el sistema educativo y sus políticas distancien las funciones de los distintos profesionales de la educación ante la búsqueda conjunta de nuevas sinergias colaborativas tan necesarias para la ardua tarea educativa actual. Esto a su vez, ha dado como resultado la acotación y construcción de barreras -por lo menos conceptualmente hablando- entre no solo la escuela y la sociedad, sino también entre los docentes y profesionales de la educación cuya acción se desvincula de la instrucción, el adoctrinamiento y la reproducción acrítica de procesos socializadores, para acercarse a la reconstrucción crítica de la ciudadanía.

Esta nueva concepción ciudadana se acoge a un desarrollo holístico que piensa en el ser humano como un todo, incorporando nuevas relaciones interpersonales en esta época postmoderna.

Sin embargo, si analizamos las propuestas educativas actuales nos damos cuenta rápidamente que éstas han quedado sujetas a perspectivas curriculares que proporcionaban respuesta a una sociedad industrial -en la que la trasmisión de conocimientos tenía como objetivo su producción y reproducción y la educación era un instrumento del mercado global-y que ignoraban las necesidades de una sociedad postmoderna como la actual, caracterizada por su complejidad e interconexión y cuya estructura social y relacional se distancian sobremanera de otras épocas.

Los espacios escolares actuales deben progresar, desvinculándose de la función de ser meros distribuidores de conocimientos (sujetos a la concepción subsidiaria del sistema económico), en el que las personas se convierten en agentes pasivos consumidoras de lo que Illich (2012) denominaba como "mito del consumo sin fin", y convirtiendo los centros educativos en un eslabón más de este ritual, en el que predominan además unos "valores envasados" que responden más a la necesidad de entender la escuela como negocio que como espacio crítico de reconstrucción conceptual en el que "significados, sentimientos y conductas de la comunidad social [se mezclan] con el desarrollo singular de las nuevas generaciones"(PérezGómez, 1997, p. 39).

2. Es por ello que a partir de ahora en este artículo no se hará distinción entre centros o espacios educativos formales o no formales, inclinándonos por la clasificación de institucionalizados o no institucionalizados. Con ello asimilamos la relación de los espacios educativos a la presencia y regulación de los órganos constitucionales de un Estado, y no según la subordinación del concepto educativo al espacio en el que desarrolla su praxis. 
Si se pretende y se lucha por la formación de ciudadanos y ciudadanas capaces de iniciar, construir y/o reconstruir un futuro en el que ellos mismos sean capaces de empoderarse, promoviendo una sociedad en la que la humanidad con sus relaciones, cobre mayor importancia que el mercado, y en la que las personas "valoran más la esperanza que las expectativas" (Illich, 2012, p. 145) no se puede eludir ni negar la necesidad de la creación de pensamientos conscientes y responsables en los que la Educación Social postmoderna fomente la construcción dialéctica de una nueva realidad educativa.

Se instaura entonces, la necesidad de establecer acciones a medio y largo plazo para el desarrollo, convirtiendo los contextos locales en educadores, incluyendo como agentes educativos activos a todas las personas que lo conforman, sus instituciones y tipos de organización; así como clarificando las funciones de cada uno de ellos.

Entender y complementar las funciones profesionales, según las características de la época, es la clave en la consecución de una nueva cultura educativa que asuma la evolución de las profesiones y de las propias relaciones interpersonales e interprofesionales.

En el caso de la Educación Social es importante resaltar la importancia de no establecer una contraposición entre la misma, como trasmisora de contenidos de cultura, y una Educación Social que permita la socialización e integración de las personas; todo lo contrario, ningún fin de la Educación Social puede obtener una socialización sin un entendimiento y aceptación de la cultura y el conocimiento. Es la praxis propia de la Educación Social lo que no se puede desvincular de cualquier espacio en el que las relaciones humanas destaquen por su pluralización, reflejo de la realidad.

Por ello, comprender las problemáticas de la sociedad actual y repensar la educación desde la necesidad de educar para la sociedad del conocimiento y no para la economía del conocimiento (Fernández-Sierra, 2011) es sin duda, uno de los caminos que es necesario emprender para avanzar hacia una sociedad caracterizada por la igualdad y la justicia social y que se fundamenta en el desarrollo personal de los individuos construido desde la libertad y la acción ${ }^{3}$. Por el contrario, desligar la Educación Social de los espacios en los que se da su praxis, es negar su concepto como derecho, así como su carácter propulsor de trasformación necesario para la satisfacción de las necesidades emergentes de la sociedad en la que vivimos.

\subsection{Un tema candente, o de los estudios sobre el tema}

En los últimos años, el re-pensamiento de la comunidad educativa y del sistema actual nos lleva, desde distintos planteamientos, a la exposición de la necesidad de un cambio tanto conceptual, como estructural de lo Educativo. Si en este sentido encontramos numerosos estudios y publicaciones, que además de exponer esta necesidad, proponen también trabajar con equipos interprofesionales (FernándezLarragueta, Fernández-Sierra y Rodorigo, 2014; Escarbajal, Arnaiz y Giménez, 2017;

\footnotetext{
3. Como actividad humana propuesta por Hanna Arendt (1997).
} 
Parrilla, Sierra y Fiuza, 2018) cuando se trata de justificar o reflexionar sobre la presencia o incorporación de Educadores o Educadoras Sociales en este ámbito, vemos acotados de forma significativa los referentes teóricos.

Para analizarlo hemos llevado a cabo una investigación bibliográfica que ha abarcado el análisis del papel de los educadores sociales en los centros educativos. La investigación ha tenido tres momentos claves: a) la búsqueda de datos. Se han consultado múltiples documentos bibliográficos recogidos en diversas bases de datos como DIALNET, WOS, SCOPUS, Google Scholar, etc. utilizando como términos de búsqueda educación social y escuela, el educador en la escuela, instituciones educativas y educadores, y permitiéndonos así recoger la información, ideas y datos sobre el objeto a estudiar; b) el análisis de contenido deductivo-inductivo (Osses, Sánchez e Ibañez, 2006). Una vez recogidos los datos, éstos se han organizado, interpretado y reestructurado, tomando anotaciones en cada uno de ellos y categorizando la información en unidades de análisis Este proceso, se abarcó en dos fases: una primera exploratoria y una segunda más específica, restringiendo la búsqueda en función de los temas emergentes. c) La presentación y discusión de los resultados. Apoyándonos en las categorías de análisis definitivas hemos ido redactando y construyendo este texto.

Aun siendo verdad que la epistemología que sustenta nuestro tópico es aún escasa, de los últimos planteamientos y estudios llevados a cabo sobre el tema, podemos relatar lo debatido agrupándolos en tres dimensiones: a) estudios que definen las bases epistemológicas de la educación social; b) investigaciones que se centran en la inclusión como eje del desarrollo humano; c) trabajos que tratan de justificar, analizar y reflexionar sobre el papel del Educador Social en las escuelas.

La primera hace referencia a los estudios (Pérez, García y Fernández, 2014; VaIlés y Pérez, 2015; Caride, Gradaílle y Caballo, 2015; Sancho, Jornet y González, 2016) que proyectan el compromiso de la Pedagogía Social ante la reciprocidad del ámbito social en la educación. Tanto desde el análisis histórico-pedagógico de la Pedagogía Social como base epistemológica de la Educación Social, como desde el estudio de la parte subjetiva o social del concepto educativo; se abren debates y planteamientos que evidencian el vínculo existente entre lo pedagógico y lo social, promoviendo el entendimiento integral del concepto educativo desde la praxis de los centros educativos institucionalizados. Especial interés para nuestro trabajo asume el estudio de Vallés y Pérez (2015) que persigue dar respuestas y mejoras a la falta de precisión y/o equívoca comprensión a la que la Educación Social debe hacer frente. Esta problemática no es ajena a nuestras instituciones, y mucho menos a las escolares, que dependen no sólo de la perspectiva genérica estatal, sino de la compresión de los órganos autonómicos y de la asunción de la cultura y concepto educativo de los propios centros.

La segunda, engloba los trabajos que plantean los principios democráticos e inclusivos (Murillo y Hernández, 2014; Belavi y Murillo 2016; Durán y Giné, 2017) como eje principal en el desarrollo personal y social, no sólo del alumnado, sino, de toda la comunidad educativa. La proyección de la consecución de una educación inclusiva desde cualquier centro y/o ámbito pedagógico, supone un objetivo 
fundamental en los replanteamientos que demandan una comprensión integral del concepto educativo. Es para la consecución de una sociedad más equitativa, justa e inclusiva que debe incorporarse en la formación de su ciudadanía el concepto democrático.

La tercera, abarca los trabajos de los últimos años (Menacho, 2013; Amador, Esteban, Cárdenas y Terrón, 2014; Ortega, 2014; Vallés y Pérez, 2015) que incitan a la justificación, análisis y reflexión de la figura del Educador o Educadora Social en los centros educativos institucionalizados y su papel en ellos. Aunque, como hemos indicado con anterioridad, los planteamientos dirigidos a la presencia de la Educación Social en estos centros se ven acotados de forma significativa, su incorporación a la comunidad educativa en algunas comunidades autónomas, así como la progresión en el entendimiento de la profesión y las constantes necesidades de entender y clarificar sus funciones según su ámbito de actuación, han iniciado la necesidad de reflexionar sobre esta situación. Aun siendo pocos y recientes resulta interesante resaltar especialmente algunos de ellos: a) el estudio de González, Olmos y Serrate (2016), que profundiza en la diferenciación y especificación de las funciones del Educador y Educadora Social en los centros de Educación Secundaria de las distintas Comunidades Autónomas; b) el artículo de Sierra, Vila, Caparrós y Martín (2017), que situándose en el contexto andaluz, supone un primer registro de un estudio profundo de la cuestión; c) el artículo de Terrón, Cárdenas y Rodríguez (2017), quienes ofrecen una relación entre Educación Social y educación inclusiva como eje natural de la cultura educativa posmoderna a través del trabajo en red. Su objetivo es conocer las funciones y competencias de esta figura profesional referente a los contextos multiculturales de los centros educativos institucionalizados andaluces, llevando a cabo una investigación desde un enfoque metodológico cuantitativo, que utiliza el cuestionario como instrumento de recogida de información; y d) el trabajo de Dapía y Fernández (2018) quienes con su estudio hacen que no dejemos de lado el análisis de la formación inicial de educadores y educadoras sociales, entendiendo el periodo de formación inicial como un espacio privilegiado de socialización y construcción de la identidad profesional.

\subsection{Replantear la práctica, o de las funciones del educador posmoderno en la escuela tradicional}

Realizando un recorrido por las competencias del perfil profesional desde un planteamiento generalizado (sin adecuación específica del ámbito de actuación), podemos ver cómo, según el documento profesionalizador de ASEDES4 (2007), estas se dividen en tres grupos: a) funciones preventivas; b) funciones mediadoras; y c) funciones educativas.

Las preventivas, persiguen la detección, valoración y prevención de necesidades socioeducativas y factores de riesgo que pueden desembocar en situaciones vulnerables. Engloban, la promoción de valores y principios sociales que promuevan la convivencia y la calidad de vida personal y grupal; programas de promoción para la

4. Asociación Estatal de Educación Social. 
salud, de inteligencia emocional; o, prevención y colaboración en el tratamiento del absentismo escolar a desempeñar con el alumnado, la familia y el resto de la comunidad educativa; las mediadoras se relacionan con la mediación de la comunidad educativa; tanto en lo referente a conflictos (intervención en el Plan de Convivencia del Centro), como en la relación y comunicación de la familia, el alumnado e instituciones implicadas en la acción educativa; y las educativas, aquellas que implican la participación, coordinación y planificación en el diseño de programas socioeducativos; orientación académica; actividades extraescolares y complementarias; aprovechamiento de recursos culturales; sesiones informativas con las familias; creación de cauces comunicativos; desarrollo y evaluación de la acción tutorial, etc. Su objetivo principal es favorecer el clima afectivo positivo, así como el desarrollo personal y comunitario.

Pero al mismo tiempo acciones, funciones o tareas que, si de por sí se incardinan perfectamente en el desarrollo de un acto educativo, entendido éste como un hecho humano complejo que se puede interpretar subjetiva y culturalmente y reinterpretar en función de la individualidad de cada uno; cuando las comparamos con los principios bases de la Ley Orgánica de Mejora de la Calidad Educativa (LOMCE) y aún a pesar de fundamentarse en distintas epistemologías y divergente implementación, nos es demasiado difícil justificar y legitimar en la escuela obligatoria de nuestro país.

De hecho, si nos paramos a revisar las funciones de los y las Educadoras Sociales en las escuelas y los principios básicos de la LOMCE (ver Tabla1) podemos ver cómo éstos se complementan, fundamentalmente debido a que: a) los principios básicos de la LOMCE se plantean desde una transversalidad que requiere de la aportación de un profesional -el educador social en este caso- que se aleje de la acción instructiva organizada en asignaturas típica de la labor docente; y b) la necesidad que se plantea desde la LOMCE, de mejorar la calidad de la educación, pasa necesariamente por replantearse una acción educativa que históricamente se ha venido desarrollando desde la individualidad del docente en el aula, pero que en la sociedad postmoderna en la que vivimos se ha quedado anacrónica.

Así, si pensamos en la calidad de la educación para todo el alumnado nos damos cuenta rápidamente de la necesidad que existe tanto de un profesional especializado en la mejora de los espacios de convivencia, como de un profesional que apoye a los docentes en su proceso formativo para llegar a ser competentes en materias educativas -que no sólo instructiva-; si hablamos de equidad que necesitamos, no sólo de personal cualificado sino también de actividades complementarias y extraescolares socioculturalmente contextualizadas que mejoren las relaciones centro-entorno y que propicien la creación de redes de comunicación estables; si lo hacemos haciendo referencia a la participación de la comunidad educativa necesitaremos de profesionales que se encarguen del diseño, la implementación y la evaluación de propuestas para fomentar las relaciones del centro con el entorno social en el que está encuadrado; etc. 


\section{Tabla 1. Elaboración propia}

\begin{tabular}{|c|c|}
\hline Principios de la LOMCE & FUNCIONES del EDUCADOR SOCIAL: \\
\hline $\begin{array}{l}\text { La calidad de la educación para todo el alumnado, indepen- } \\
\text { dientemente de sus condiciones y circunstancias }\end{array}$ & $\begin{array}{l}\text {-. Apoyo a la formación del profesorado. } \\
\text {-. Creación de espacios de convivencia. }\end{array}$ \\
\hline $\begin{array}{l}\text { La equidad, que garantice la igualdad de oportunidades } \\
\text { para el pleno desarrollo de la personalidad a través de la } \\
\text { educación, la inclusión educativa, la igualdad de derechos } \\
\text { y oportunidades que ayuden a superar cualquier discrimi- } \\
\text { nación y la accesibilidad universal a la educación, y que } \\
\text { actúe como elemento compensador de las desigualdades } \\
\text { personales, culturales, económicas y sociales, con especial } \\
\text { atención a las que se deriven de cualquier tipo de disca- } \\
\text { pacidad. }\end{array}$ & $\begin{array}{l}\text {-. Apoyo a la formación del profesorado. } \\
\text {-. Planificación, coordinación y desarrollo con el departa- } \\
\text { mento de actividades complementarias y extraescolares } \\
\text { o con el AMPA o con el consejo escolar, actividades so- } \\
\text { cioculturales contextualizadas que mejoren las relacio- } \\
\text { nes centro-entorno y que propicien la creación de redes } \\
\text { de comunicación estables. }\end{array}$ \\
\hline $\begin{array}{l}\text { La transmisión y puesta en práctica de valores que favorez- } \\
\text { can la libertad personal, la responsabilidad, la ciudadanía } \\
\text { democrática, la solidaridad, la tolerancia, la igualdad, el res- } \\
\text { peto y la justicia, así como que ayuden a superar cualquier } \\
\text { tipo de discriminación. }\end{array}$ & $\begin{array}{l}\text { Desarrollo de programas de interrelación con la comunidad } \\
\text { de aprovechamiento y conocimientos de los recursos del en- } \\
\text { torno, laborales, naturales, etc. }\end{array}$ \\
\hline $\begin{array}{l}\text { La orientación educativa y profesional de los estudiantes, } \\
\text { como medio necesario para el logro de una formación per- } \\
\text { sonalizada, que propicie una educación integral en conoci- } \\
\text { mientos, destrezas y valores. }\end{array}$ & $\begin{array}{l}\text { Colaboración en el desarrollo de programas de educación } \\
\text { para la salud, medio ambiente, consumo en la tolerancia e } \\
\text { igualdad, paz y otros que determinen el Dpto. de Orienta- } \\
\text { ción o el equipo directivo u otros órganos. }\end{array}$ \\
\hline El esfuerzo individual y la motivación del alumnado. & ones de absentismo esco- \\
\hline $\begin{array}{l}\text { El esfuerzo compartido por alumnado, familias, profesores, } \\
\text { centros, Administraciones, instituciones y el conjunto de la } \\
\text { sociedad. }\end{array}$ & $\begin{array}{l}\text {-. Desarrollo de programas para prevenir y afrontar la con- } \\
\text { flictividad escolar. Programas de integración escolar. } \\
\text {-. Programación y ejecución con el Dpto. de orientación } \\
\text { y el equipo directivo actuaciones encaminadas a garanti- } \\
\text { zar la información puntual a las familias sobre el proceso } \\
\text { educativo de sus hijos. }\end{array}$ \\
\hline $\begin{array}{l}\text { La participación de la co } \\
\text { ción, gobierno y funcion }\end{array}$ & $\begin{array}{l}\text {-. Diseño, implementación y evaluación de propuestas } \\
\text { para fomentar las relaciones del centro con el entorno } \\
\text { social en el que está encuadrado. } \\
\text {-. Colaboración con los órganos del centro en el cumpli- } \\
\text { miento de las normas del centro y de su reglamento de } \\
\text { régimen interno. }\end{array}$ \\
\hline $\begin{array}{l}\text { La educación para la prevención de conflictos y la resolución } \\
\text { pacífica de los mismos, así como para la no violencia en } \\
\text { todos los ámbitos de la vida personal, familiar y social, y en } \\
\text { especial en el del acoso escolar. }\end{array}$ & $\begin{array}{l}\text {-. Creación de espacios y de equipos de trabajo media- } \\
\text { dores y negociadores para la eficacia en la resolución } \\
\text { de conflictos. } \\
\text {-. Elaboración y evaluación de propuestas de programas de } \\
\text { convivencia en el centro educativo. }\end{array}$ \\
\hline $\begin{array}{l}\text { El desarrollo, en la escuela, de los valores que fomenten la } \\
\text { igualdad efectiva entre hombres y mujeres, así como la pre- } \\
\text { vención de la violencia de género. }\end{array}$ & $\begin{array}{l}\text { Seguimiento y colaboración en la respuesta educativa al } \\
\text { alumno con comportamientos problemáticos. } \\
\text {-. Programación de información, orientación y asesora- } \\
\text { miento y asociación a los alumnos. }\end{array}$ \\
\hline
\end{tabular}


Así mismo, muchos autores (San Fabian, 2006; López, 2007; Fernández-Larragueta, Fernández-Sierra y Rodorigo, 2014; Fernández-Sierra, 2017; Escarbajal, Arnaiz y Giménez, 2017; Parrilla, Sierra y Fiuza, 2018) cuando hablan de la educación como del desarrollo integral de los seres humanos reconocen la necesidad del trabajo colaborativo para su desempeño. Sin embargo, bien por falta de tiempo; bien por escasez en la formación específica, los docentes necesitan cada vez más de un profesional que, al no tener que desempeñar tareas docentes como sería el caso del educador social, pueda planificar y coordinar la labor interdisciplinar y transversal que desde la legislación se plantea.

\section{LA EDUCACIÓN SOCIAL EN LA ESCUELA COMO PRÁCTICA EN CONSTRUCCIÓN, O DE LA VARIABILIDAD DE LA ACCIÓN}

Los y las educadoras sociales son entonces hoy en día necesarios para que los centros educativos puedan ofrecer una educación de calidad, entendida ésta desde la perspectiva de la acción y el análisis de las necesidades reales de cada entorno ya que negar su presencia en estas instituciones, significaría negar las propias relaciones humanas que en ellas se dan y que desembocan en la formación de la persona.

Sin embargo, las experiencias y extractos normativos que relatan las controversias, incongruencias y problemáticas relacionadas con la incorporación de educadores y educadoras a los centros educativos, así como la variabilidad normativa que define la labor de los y las educadoras sociales en nuestro país dejan patente la variabilidad de la acción y su inestabilidad frente a la sociedad como profesión encargada de dichos quehaceres.

En primer lugar, lo escrito sobre su incorporación muestra que siempre que se analiza el perfil del educador social ligando su acción a contextos vulnerables y/o marginales y no al centro educativo como tal y a la ciudadanía en general, perdiendo de vista la necesidad de entender al educador social como un agente de cambio que se encarga de que todos los ciudadanos se enfrenten con las mismas herramientas a la vida del espacio público democrático.

En segundo lugar, su incorporación a los centros educativos se define fundamentalmente de dos formas distintas según recorremos la geografía española. Por un lado, nos encontramos con espacios en los que la incorporación de educadores y educadoras se ha planteado incluyendo al educador social de forma constituyente en la estructura organizativa de los centros, como es el caso de Comunidades Autónomas como Castilla la Mancha (2002), Extremadura (2002) y Andalucía (2006); o por otro, limitando su presencia a intervenciones "con programas o proyectos específicos... en el ámbito educativo a través de servicios sociales, asociaciones, empresas, ayuntamientos, etc." (Terrón-Caro, Cárdenas-Rodríguez y Rodríguez-Casado, 2017, p. 28) como en las comunidades de Aragón, Canarias, Castilla y León, País Vasco, Valencia, Cataluña, Galicia, Islas Baleares, Madrid o Navarra5.

5. Cabe destacar además aquí que en los momentos que estamos escribiendo este texto se están poniendo en marcha acciones novedosas en las Islas Baleares y Canarias, donde se han aprobado la incorporación y/o recuperación de Educadores y Educadoras Sociales para el curso 2017/2018 bajo programas 
Sin embargo, entender la Educación Social como motor y herramienta de trasformación, así como impulsora de la acción inclusiva de los colectivos vulnerables, implica la comprensión de dos elementos a la vez contradictorios y concurrentes. Por un lado, es necesario alejar la profesionalización del educador social en la escuela de una conceptualización puntual y aislada de la educación institucional, para que no acabe entendiéndose como una tarea especializada que mira tan solo a la resolución de problemas concretos desde un enfoque asistencialista y remedial que ha definido en otros momentos perfiles profesionales que han entrado a los centros educativos como por ejemplo los psicopedagogos o los mediadores interculturales (Fernández-Sierra y Fernández-Larragueta, 2006; Santana, y Feliciano, 2006; Fernández-Larragueta, 2011; Rodorigo, 2013; Fernández-Larragueta, Fernández-Sierra y Rodorigo, 2014); y por otro, acercándola cada vez más a una perspectiva integral y holística más propias de comunidades que también educan.

Es además vital incorporar la figura del Educador/a Social en todos los centros educativos institucionalizados, dado que esto podría significar: la definición de sus funciones por parte de los órganos reguladores legislativos y el reconocimiento de "dos condiciones [fundamentales] cuando hablamos de sus funciones. Por un lado, ser coherentes con una determinada línea de intervención y actividades, con criterios de continuidad y claridad de actuación. Por otro, estar permanentemente integradas y reconocidas respecto al resto de actuaciones y funciones del personal docente."(Galán y Castillo, 2008, p. 126) Algo que supondría, además la ruptura del pensamiento asignaturizado que define la escuela alejando el papel del educador social del de mercante educativo, que desembarca en las escuelas como comerciante de programas o proyectos externos.

Finalmente recordar que no todos los espacios escolares poseen las mismas características, ni que todos los profesionales entienden la Educación Social posmoderna que desde aquí se defiende de la misma forma, por lo que su incorporación, asimilación y definición de funciones, deben ser propulsadas desde el compromiso de toda la estructura organizativa escolar. Si algo queda claro, es que para poder concretar las funciones del Educador/a Social en los centros educativos, se necesita una ruptura con la perspectiva asistencial y complementaria con la que hoy día se le está tratando, abandonando los discursos hegemónicos, caracterizados por la jerarquización de las macro estructuras educativas que los caracteriza.

\subsection{El educador social como profesional interno, o de la necesidad de repensar la colaboración interprofesional}

Acabar con la perspectiva que presupone al Educador/a Social como un mitigador de problemáticas, supone crear una línea de intervención continua, clara y coherente en sus funciones, así como, que se inicie un entendimiento de la educación desde un proceso integral que debe ser llevada a cabo, no de forma puntual o especializada, sino a través de un escenario común entre todos sus agentes. Trabajar desde, por y para un proyecto educativo común supone el reconocimiento de cada una de las

que promueven la convivencia en el centro escolar y su entorno (curso actualmente en desarrollo). 
partes que compone la comunidad, facilitando una línea de trabajo con otras instituciones fuera de los "muros escolares institucionalizados" (familias, asociaciones, centros comunitarios, etc.) y evitando la dualidad e inmovilidad del concepto de educación que tanto está obviando las necesidades de un mundo expuesto a constantes cambios.

Es necesario de hecho, repensar la estructura organizativa, que como indican Fernández-Larragueta, Fernández-Sierra y Rodorigo (2014), supere la superficialidad de las relaciones interprofesionales hacia una concepción conjunta del pensamiento docente, alejando a éste de la instrucción e individualización y permitiendo, así, la concreción de las funciones de sus profesionales a partir de un proyecto educativo común.

Y es que si se pretende abandonar la idea de una educación tradicional sujeta a perspectivas curriculares propias de la época industrial/moderna (Fernández-Larragueta, Fernández-Sierra y Rodorigo, 2017) es ineludible impulsar el trabajo de forma conjunta y coordinada, en aras de aprovechar el potencial que tienen los escenarios educativos como espacios para mejorar la actitud crítica de las futuras generaciones (Hargreaves, 2005; San Fabian, 2006; López, 2007).

En los últimos años, esta incorporación de nuevas figuras profesionales especializadas en los centros educativos ha sido un intento de respuesta a las necesidades emergentes y a los nuevos contextos que se dan en ellos que, sin embargo, han dejado patente la falta de coordinación como elemento trasformador de la educación, pues la acción educativa promovida no se ha repensado hacia una visión y práctica educativa, sino a una asignaturización de las funciones de cada profesional (Rodorigo, 2013).

Así, si se intenta concretar las funciones del Educador o Educadora Social desde una perspectiva que lo asuma como agente externo desligado del currículum, no se hace nada más que repetir el patrón ya experimentado desde los años 90 con la figura del psicopedagogo en los centros educativos, como ya planteaban hace una década Fernández-Sierra y Fernández-Larragueta (2006) en su estudio. Se hace necesario romper con la resistencia al cambio que caracteriza al sistema educativo y que estandariza los perfiles profesionales especializados. Si la trasformación del concepto de educación necesita una reestructuración, esto mismo, a su vez, no puede darse sin una reconstrucción en la organización profesional que abandone la idea de cooperación en base a un currículum estático que jerarquiza, de forma natural, el compromiso y las relaciones entre todos los agentes involucrados.

Aunque se vaya asumiendo la necesidad de una cultura común profesional en los centros educativos institucionalizados, es necesario enfocarla hacia la "revolución cultural" que propone Bauman (2013), pues sin esa pretensión es imposible encaminarse hacia proyectos educativos compartidos que persigan la acción inclusiva y de trasformación social que incluya a sus agentes como generadores de sus propios contextos.

La praxis dependiente a los contextos de actuación que presentan los Educadores Sociales supone cierta complejidad en la concreción de sus funciones específicas, 
pues no pueden estar subordinadas a una percepción legislativa externa o incluso a una percepción de los propios compañeros de centro. Es entonces primordial un concepto educativo que persiga una coordinación que respalde la acción de todos sus agentes, lo cual, requiere de espacios públicos para ello.

Impulsar el ámbito de actuación de la Educación Social dentro del espacio escolar, ayudaría a la concreción de las funciones según las necesidades de cada contexto. Se trata entonces de entender y aceptar esta nueva figura como agente de trasformación de la estructura organizativa, alejándola de una percepción de tropa auxiliar $^{6}$, solucionadora o facilitadora de las funciones de otros profesionales (que no desarrollan por sobrecarga burocrática o falta de tiempo). Se trata de proporcionar su propio espacio en el centro escolar que permita el desarrollo de sus funciones, no quedando éstas sujetas a las carencias existentes según el centro o la Comunidad Autónoma.

\section{Conclusiones no Conclusivas, O de la NeCESidAd de SEguiR PENSANDO}

Llegados a este punto y para concluir este trabajo vamos a dejar constancia de unas ideas claves que han ido emergiendo de la investigación bibliográfica llevada a cabo, en aras de seguir investigando y reflexionando sobre la construcción de una profesión pedagógica que debe ser incorporada a las instituciones educativas institucionalizadas para hacer frente a la actual sociedad posmoderna.

En este sentido consideramos interesante subrayar unas ideas que sin duda aportan una nueva visión al debate epistemológico sobre la necesidad de la entrada de educadores y educadoras sociales a los centros educativos españoles, no como expertos que acuden puntualmente sino como parte del equipo educativo del centro.

En relación al análisis de las funciones del educador social en las escuelas decir que:

a) Es urgente definir colegiadamente y dialécticamente las funciones de la educación social en la escuela. A pesar de que exista un libro blanco de la Pedagogía y la Educación Social que define las bases de la profesión del educador social; Colegios Profesionales organizados por Comunidades Autónomas en cuestión de funcionamiento y agrupados a nivel estatal a través del Consejo General de Colegios Profesionales (CGCEES) que trabajan día a día para construir y reconstruir desde la práctica las bases de la acción; así como múltiples espacios virtuales como blog y/o páginas web desde los que se lucha para la visibilización de la profesión; todavía no existe un acuerdo nacional sobre las funciones a desarrollar por parte del educador o educadora social en la escuela. Esto, debido por un lado a la falta de institucionalización del perfil del educador social en la escuela; y por otro, a la joven trayectoria de la misma, provoca diversos desajustes a la hora de entender el papel de estos profesionales en las escuelas: 1) como muestran otros estudios llevados a cabo en distintos ámbitos (Fernández-Larragueta, Fernández-Sierra y Rodorigo, 2014, 2017), las Comunidad Autónoma acaban legislando de forma local, olvidando el carácter

6. El ejército romano, compuesto íntegramente por ciudadanos romanos, se nutría del apoyo y auxilio militar de unidades de combate compuestas por ciudadanos no romanos. 
global de la función del educador social, primando programas o proyectos puntuales a poner en marcha en las escuelas, frente a la acción coordinada de un profesional que actúa desde dentro y redefiniendo cada vez sus funciones en función del marco de acción en el que se incardinan; y 2) socialmente, no se entiende bien cuál sería su papel en las escuelas y por lo tanto no se lucha para su incorporación como miembro del claustro. Finalmente, plantear su incorporación en la comunidad educativa, ya sea como miembro de los órganos colegiados o como parte del departamento de orientación junto con orientadores y maestros y maestras de ATAL, se hace necesario para poder ratificar el sentido tanto de la profesión, en cuanto a términos de igualdad se refiere; como a la correcta comprensión de la Educación Social y la Calidad Educativa, tan demandada en los últimos tiempos y objetos de evaluaciones continuas. De hecho, el desconocimiento de las potencialidades de este profesional y la inexactitud de su cumplimiento en la integración en los centros educativos de nuestro país, provoca por un lado, que la profesión siga sin definirse epistemológicamente; y por otro, que se dejen de atender de forma integral cuestiones como la lucha contra el racismo, la injusticia, el bullying y el ciberbullying; la búsqueda del respeto mutuo, la empatía y la educación; la puesta en marcha de programas de prevención contra el maltrato por cuestiones de género o animal, etc.

b) Necesitamos educadores posmodernos, para escuelas posmodernas. La sociedad ha cambiado y los valores que la sustentan también lo han hecho. Sin embargo, la escuela no ha cambiado. Incluso cuando nos paramos a reflexionar sobre la necesidad de educadores y educadoras sociales en las escuelas sabedores de que los docentes ya no pueden enfrentarse solos a una tarea que es cada día más cambiante, seguimos reflexionando utilizando como espacio ecológico de desarrollo, centros educativos que se organizan y estructuran como hace cientos de años, que se autodefinen exclusivamente en función de su misión instructiva como si el concepto de maestro y maestra tan solo tuviera sentido unido unilateralmente al de clase magistral. Lamentablemente, este inmovilismo que define la macroestructura escolar acaba definiendo a su vez, la acción de otros profesionales que entran a las escuelas. Profesionales nuevos (Fernández-Sierra y Santos, 2014; González, Llamas y Correa, 2016; Fernández-Sierra, 2017) con funciones y acciones tradicionales que en vez de responder a las demandas de la nueva sociedad, reproducen rutinas como si de otros docentes más se tratara. Sin embargo, es necesario que cambiemos. Hay que resaltar la importancia de la creación de espacios educativos que no solo busquen la transmisión acrítica de contenidos académicos seleccionados por terceros (Torres, 1993, 2008), sino que primen el desarrollo integral de los seres humanos, frente justamente a la objetivación y asignaturización típica de estos últimos tiempos.

En relación a la posibilidad de acción del educador social en las escuelas tanto en su perspectiva relacional como de funcionamiento, decir que:

c) La colaboración interprofesional favorece el potencial que tienen los agentes de integración en las escuelas. Invertir en recursos humanos y materiales sin que estos acaben siendo relevantes en el espacio en los que actúan es sin duda algo que se aleja sobremanera de la idea de una educación de calidad para todos. Sin embargo, como podemos ver de otras investigaciones (Rodorigo, 2013; Fernández-Larragueta, 
Fernández-Sierra y Rodorigo, 2014; González, Llamas y Correa, 2016) tradicionalmente los profesionales que han entrado a las escuelas lo han hecho de puntilla, desarrollando su labor al margen de los demás y del Proyecto Educativo de Centro, considerándose como profesionales expertos que intervienen en situaciones complejas y puntuales. Esta percepción es algo que, sin duda, ha favorecido la administración al proponer/imponer, una y otra vez, cambios organizativos y de trabajo en los centros escolares, estableciendo órganos de coordinación formales y estructurados que provocan que los profesionales de la educación, obligados legislativamente a colaborar con otros, dan pie a un tipo de coordinación que, lejos de representar un momento para el debate y la reconstrucción de la práctica, es potenciadora de una colaboración simulada que aquieta la oportunidad de establecer cambios reales que lleven al profesorado hacia la construcción de un entorno educativo holístico y de calidad (Hargreaves, 1996; Fullan y Hargreves, 1997).

d) La supra-estructura organizativa y legislativa de los centros de educación institucionalizada inhiben el potencial del trabajo colaborativo. Que el profesorado entienda la colaboración en los centros escolares como un espacio legislativo en el que tratar los aspectos más formales del funcionamiento del centro, provoca que cuando hablamos de la colaboración con un agente como en este caso el educador o educadora social, los docentes ni siquiera aspiren a ello, ya que en principio no está establecido normativamente. Sin embargo, tan solo podremos sumar esfuerzos cuando éstos se hagan desde un planteamiento común, de toma de decisiones dialécticas y en igualdad de oportunidades. Es necesario que antes de la Ilegada de estos profesionales se trabaje en los centros el tema de la coordinación interprofesional entendida como un proceso interactivo que necesita de una comunicación fluida, a través del que, profesionales de la educación, sin perder sus especificidades y su creatividad, en un plano de igualdad, buscan de forma conjunta disponer elementos metódicamente o concertar medios para buscar un objetivo común, identificar problemas educativos, plantear mejores programas, estrategias de aprendizaje y contenidos significativos para todo el alumnado, además de intentar desarrollar prácticas educativas más ventajosas y holísticas, favoreciendo una reestructuración del sistema educativo, haciendo en la práctica interactuar y acoplar un determinado flujo de acciones (Rodorigo, 2015, p. 27); en aras, fundamentalmente de sumar en lugar de restar.

\section{REFERENCIAS BIBLIOGRÁFICAS}

Amador, L. V., Esteban, M., Cárdenas, M. R. y Terrón, M. T. (2014). Ámbitos de profesionalización del educador/a social: perspectivas y complejidad. Revista de $\mathrm{Hu}$ manidades, 21.

Arendt, H. (1997). ¿Qué es la política? Buenos Aires, Argentina: Paidós.

ASEDES (2007). El educador y la educadora social en el estado español: una concreción de su trabajo en centros escolares. Recuperado de: http://webcache.googleusercontent.com/search?q=cache:YP_JhGFLFDsJ:www.eduso.net/archivos/ESCE. $\mathrm{doc}+\mathrm{ycd}=1 \mathrm{yhl}=\mathrm{esyct}=\mathrm{clnkygl}=\mathrm{es}$.

Bauman, Z. (2003). Modernidad líquida. Buenos Aires, Argentina: Fondos de Cultura. 
Bauman, Z. (2013). Sobre la educación líquida en un mundo líquido: conversaciones con Ricardo Mazzeo. Buenos Aires, Argentina: Paidós.

Belavi, G. y Murillo F. J. (2016). Educación, democracia y justicia social. Revista Internacional de educación para la Justicia Social (RIEEJS), 1(5), 13-34. http://doi. org/10.15336/riejs2016.5.1.

Caride, J. A., Gradaílle, R. y Caballo, M. B. (2015). De la pedagogía social como educación, a la educación social como pedagogía. Perfiles Educativos, 37, 148, 4-11.

Dapía, M. D. y Fernández, M. R. (2018). Educación Social y escuela en España. A propósito de la formación e inserción laboral. Revista iberoamericana de Educación, 76, 209-228.

Durán, D. y Giné, C. (2017). La formación del profesorado para la educación inclusiva: un proceso de desarrollo profesional y de mejora de los centros para atender la diversidad. Revista Latinoamericana de Educación Inclusiva, 2(5), 153-170.

Escarbajal, A., Arnaiz, P. y Jiménez, A. (2017). Evaluación de las fortalezas y debilidades del proceso educativo en centros de infantil, primaria y secundaria desde una perspectiva inclusiva. Revista Complutense de educación, 2(28), 427-443. http:// doi.org/10.5209/rev_RCED.2017.v28.n2.49423.

Fernández-Larragueta, S. (2011). La formación inicial en la historia profesional de los psicopedagogos noveles en Institutos de Educación Secundaria (IES). Revista de Educación, 354, 529-547.

Fernández-Larragueta, S. Fernández-Sierra, J. y Rodorigo, M. (2014). Coordinación interprofesional en los centros educativos: una apuesta para la inclusión. ESE: Estudios sobre educación, 27, 193-211.

Fernández-Larragueta, S. Fernández-Sierra, J. y Rodorigo, M. (2017). Expectativas socioeducativas de alumnas inmigrantes: escuchando sus voces. Revista de investigación educativa, RIE, 35(2), 483-498.

Fernández-Sierra, J. (2011). Formar para la economía del conocimiento vs educar para la sociedad del conocimiento: una visión desde la pedagogía. Málaga: Aljibe.

Fernández-Sierra, J. (2017). Alumnado inmigrante en la ESO: vulnerabilidad pedagógica del sistema educativo. Educación XX1, 20(1), 121-140. http://doi.org/10.5944/ educXX1.12855.

Fernández-Sierra, J. y Fernández-Larragueta, S. (2006). La construcción del conocimiento profesional y la socialización de los psicopedagogos/as de centros noveles. Educación, 341, 419-440.

Fernández-Sierra, J. y Santos, G. (2014). Orientar las transiciones del alumnado inmigrante: más que un reto multiprofesional. REOP, 25(2), 8-23.

Freire, P. (2012). Pedagogía del oprimido (2. edición). Madrid: Siglo XXI España.

Fullan, M. y Hargreaves, A. (1997). ¿Hay algo por lo que merezca la pena luchar en la escuela? Morón (Sevilla). M.C.E.P. 
Galan, D. y Castillo, M. (2008). El papel de los educadores sociales en los centros de secundaria: una propuesta para el debate. Educación Social, 38, 121-133.

Gómez-Serra, M. (2003). Aproximación conceptual a los sectores y ámbitos de intervención de la educación social. Pedagogía social, 2(10), 233-251.

González, I. Llamas, C. y Correa, R. (2016). El orientador escolar en contextos de diversidad cultura. Un análisis de su práctica. Cultura y Educación, 28(2), 426-433.

González, M.; Olmos, S. y Serrate, S. (2016). Análisis de la práctica profesional del educador social en centros de educación secundaria. Pedagogía Social. Revista Interuniversitaria, 28, 229-243.

Hargreaves, A. (2005). Profesorado, cultura y postmodernidad. Madrid: Morata.

Illich, I. (2012) La sociedad desescolarizada. Buenos Aires: Godot.

Lipovetsky, G. (2003). Metamorfosis de la cultura liberal. Barcelona: Anagrama.

López, A. (2007). 14 ideas clave: El trabajo en equipo del profesorado. Barcelona: Graó.

Lyotard, J. (1979). La condición postmoderna. Madrid: Cátedra.

Menacho, S. (2013). El Educador Social en la escuela. Justificación de la necesidad de la educación Social en la escuela. Revista de Educación Social, 16. Recuperado de http://www.eduso.net/res/pdf/16/eduesc_res_16.pdf.

Ministerios de Educación, Cultura y Deporte (2013). Ley Orgánica para la Mejora de la Calidad Educativa. Publicada en BOE №295, martes 10 de diciembre de 2013. España.

Murillo, F. y Hernández, R. (2014). Liderando escuelas justas para la justicia social. Revista Internacional de Educación para la Justicia Social (RIEEJS), 3(2), 13-32.

Ortega, J. (2014). Educación social y enseñanza: los educadores sociales en los centros educativos, funciones y modelos. Edetania, 45, 11-31.

Osses, S., Sánchez, I. y Ibañez, F. (2006). Investigación cualitativa en educación. Hacia la generación de teoría a través del proceso analítico. Estudios Pedagógicos, 32(1), 119-133.

Parrilla, A., Sierra, S. y Fiuza, M. (2018). Lecciones esenciales sobre el trabajo en red inter-escolar. Profesorado. Revista de Currículum y Formación de Profesorado, 22(2), 51 69. http://hdl.handle.net/10481/53078.

Pérez-Gómez, A. I. (1997). Ensayo de pedagogía crítica. Madrid, España: Editorial Popular.

Pérez, G. García, J.L y Fernández, A. (2014). Fundamentos de la Pedagogía y de la Educación Social. Interfaces científicas-Educação, 1(3), 21-32.

Porras, R., García, M. y Cotrina, M. (2009). Posibilidades y límites del programa ATAL en el marco de una escuela inclusiva. El caso de la provincia de Cádiz. Revista Educación inclusiva, 2(1), 11-28. 
Rodorigo, M. (2013). Mediación y colaboración interprofesional para la educación intercultural. Estudio de caso. Almería: Universidad de Almería.

Rodorigo, M. (2015) Postmodernidad y Escuela. Revista Vozes dos Vales: Publicações Acadêmicas, 7(4).

Rodorigo, M. (2017). De lo probablemente medible a la posibilidad de una mejora real. Los educadores sociales como garantes de la calidad socioeducativas. En Requeijo Rey, P. y Vega Baeza, R. La universidad y nuevos horizontes del conocimiento. Madrid: Tecnos.

San Fabián, J. L. (2006). La coordinación docente: Condiciones organizativas y compromiso profesional. Participación Educativa, 3, 6-10.

Sancho, C., Jornet, J. M. y González, J. (2016). El constructo Valor Social de la Educación: validación cruzada entre profesorado de escuela y universidad. Revista de Investigación Educativa, 2(34), 329-350. http://doi.org/10.6018/rie.34.2.226131.

Sierra, J. E., Vila, E. S., Caparrós, E. y Martín, V. M. (2017). Rol y funciones de los educadores y las educadoras sociales en los centros educativos andaluces. Análisis y reflexiones. Revista Complutense de educación, 2(28), 479-495. http://doi. org/10.5209/rev_RCED.2017.v28.n2.49542.

Terrón, T., Cárdenas, R. y Rodríguez, R. (2017). Educación intercultural inclusiva. Funciones de los/as educadores/as sociales en instituciones educativas. Pedagogía social, 3(29), 25-40. http://doi.org/10. SE7179/PSRI_2017.29.02.

Torres, J. (1993). Las culturas negadas y silenciadas en el curriculum. Cuadernos de Pedagogía, 217, 60-66.

Torres, J. (2008). Obviando el debate sobre la cultura en el sistema educativo: cómo ser competentes sin conocimientos. En Gimeno-Sacrístan, J., Educar por competencias, ¿qué hay de nuevo? (pp. 143-175). Madrid: Morata.

Vallés, J. y Pérez, R. (2015). Las funciones del educador social: validación del cuestionario CFES-R. Bordón, 67(2), 131-153. http://doi.org/10.13042/Bordon.2015.67209. 